
sociologie des sciences : des débats, un réexamen

\title{
Émotions et rationalité dans la sociologie classique : les cas de Weber et Durkheim
}

Charles-Henry Cuin

\section{(2) OpenEdition}

Journals

Édition électronique

URL : http://journals.openedition.org/ress/658

DOI : $10.4000 /$ ress.658

ISSN : 1663-4446

Éditeur

Librairie Droz

Édition imprimée

Date de publication : 1 juillet 2001

Pagination : 77-100

ISBN : 2-600-00610-9

ISSN : 0048-8046

Référence électronique

Charles-Henry Cuin, «Émotions et rationalité dans la sociologie classique : les cas de Weber et Durkheim », Revue européenne des sciences sociales [En ligne], XXXIX-120 | 2001, mis en ligne le 14 décembre 2009, consulté le 23 avril 2019. URL : http://journals.openedition.org/ress/658 ; DOI : 10.4000/ress.658 


\section{Charles-Henry CUIN}

\section{ÉMOTIONS ET RATIONALITÉ DANS LA SOCIOLOGIE CLASSIQUE : LES CAS DE WEBER ET DURKHEIM}

Les débats sur la rationalité de l'action occupent, en sociologie, une place centrale. Le fait n'est pas nouveau puisque cette discipline s'est en partie édifiée en référence à l'économie classique, soit pour s'y opposer (Durkheim), soit pour s'en distinguer (Pareto), soit encore pour en élargir la portée (Weber). Les diatribes durkheimiennes contre l'individualisme et l'utilitarisme du paradigme économiste, tout comme le projet paretien de réserver à la sociologie l'étude des actions non rationnelles, sont fondatrices d'une sociologie refusant de réduire l'action à sa dimension instrumentale et choisissant de lui consacrer les formes les moins réflexives de l'action. Quant à l'entreprise weberienne, elle se caractérise par une tentative de réduire - méthodologiquement s'entend - l'analyse d'une action sociale reconnue comme généralement peu ou non rationnelle aux diverses dimensions d'une rationalité purement idéal-typique. Plus généralement, la sociologie classique, qu'elle soit holiste ou individualiste, a partagé le projet commun de rechercher les ressorts de la conduite humaine dans la voie étroite entre les «passions » et les « intérêts » ${ }^{1}$.

Naguère, ces débats ont été vigoureusement ranimés par le succès des théories anglo-saxonnes du rational choice ${ }^{2}$. Leur moindre mérite n'a pas été de permettre aux théoriciens de la sociologie, voire aux philosophes, de «revisiter» la notion de rationalité, d'en montrer la complexité et la polysémie, et d'en proposer des conceptualisations plus précises et plus fécondes ${ }^{3}$. Pourtant, si ces débats ont permis à certains courants d'affirmer leurs divergences par rapport à une conception strictement rationaliste de l'action sociale et de développer des paradigmes plus ou moins concurrents, ils ont aussi largement négligé une dimension constitutive de la conception dite «classique » de l'action - à savoir la dimension proprement émotionnelle.

Cette dimension, qui paraît être le symétrique opposé de celle de rationalité, est pourtant omniprésente chez les auteurs du tournant du siècle dernier. Elle apparaît - ou transparaît - en effet sous des formes lexicales diverses: l' «effusion» (communautaire) chez Tönnies; l'action «affectuelle» [affektuel] ou le «charisme» chez Weber; les «résidus» chez Pareto; ou encore, évidemment,

Albert O. Hirschman, Les Passions et les intérêts [1977], Paris, P.U.F., 1980.

Bertrand Saint-Sernin et al. (Éd.), Les Modèles de l'action, Paris, P.U.F., 1993 (Cf., en part., Pierre Demeulenaere, Les ambiguiités constitutives du choix rationnel, Ibid., p. 51-66).

Cf., en part., Jon Elster et A. Hylland (Éd.), Foundations of Social Choice Theory, Cambidge, Cambridge Univ. Press, 1986 et James Coleman, Foundations of Social Theory, Cambridge/London, The Belknap Press of Harvard Univ. Press, 1990. 
l' «effervescence collective» ou les «états forts de la conscience collective» chez Durkheim qui, par ailleurs, utilise très souvent le terme même d' 'émotion». On n'oubliera pas non plus les belles pages consacrées par Simmel à l'analyse de certaines formes émotionnelles de l'interaction, telles que la jalousie ou encore la haine. ${ }^{4}$ Selon les cas et les auteurs, l'émotionnel s'oppose à l'une ou l'autre des caractéristiques générales du rationnel, c'est-à-dire à ce qui est réflexif, calculé, contrôlé, réglementé, prévisible. Pour autant, ce signifié générique ne fait nulle part l'objet d'une quelconque conceptualisation spécifique, comme si les termes mêmes d'émotion ou d'émotionnel, dépouvus d'ambiguïté, pouvaient être simplement renvoyés au sens commun et courant. Et c'est sans doute la raison pour laquelle une notion centrale de la sociologie classique est restée absente du lexique sociologique et, a fortiori, des débats théoriques et conceptuels de la discipline $^{5}$ - à la notable exception près des travaux de N. Elias sur le «contrôle des émotions $»^{6}$.

Notre objet principal n'est pas évidemment pas de remédier à cette absence (qui ne constitue peut-être même pas une lacune) en tentant de forger un concept sociologique de l' "émotion». Il est plutôt d'analyser le statut de la dimension émotionnelle de l'action dans la sociologie classique à travers l'œuvre de deux auteurs que beaucoup de traits distinguent, voire semblent opposer, dans ce domaine et plus généralement dans celui de la nature de l'action sociale - et qui sont donc largement représentatifs des principales orientations épistémologiques et théoriques de la sociologie classique ${ }^{7}$. Ainsi, si la première partie de ce texte est consacrée à un essai de définition de l'émotion, c'est plus par souci méthodologique que proprement conceptuel. La tâche d'identifier et d'évaluer le statut d'une notion chez des auteurs où celle-ci n'est pas indexée théoriquement requiert en effet de disposer d'une définition préalable. Par ailleurs, une telle définition sera d'autant plus opératoire qu'elle sera, d'une part, assez large pour englober le plus grand nombre de formes empiriques possibles de son objet et, d'autre part, assez spécifique pour s'appliquer pertinemment aux domaines de l'action et de la vie sociales.

\section{I - QUESTIONS DE MÉTHODE}

Le terme d' «émotion» n'étant pas un concept sociologique classique, il est moins question ici de rechercher la signification que des auteurs ont pu donner à ce terme (ou à des termes proches) lorsqu'ils l'ont employé que de rechercher comment ces auteurs ont appréhendé et, éventuellement, conceptualisé la réalité empirique particulière que l'on désigne couramment sous ce terme. Toutefois,

Georg Simmel, Sociologie. Études sur les formes de socialisation [1908], Paris, P.U.F., 1999.

On se référera avec profit à la bibliographie établie par Cléopâtre Montandon, Processus de socialisation et vécu émotionnel des enfants, Revue française de sociologie, XXXVII-2, 1999, p. 263285.

$6 \quad$ Norbert Elias, La Civilisation des mœurs [1936], Paris, Calmann-Lévy, 1973.

7 Monique Hirschhorn et Jacques Coenen-Huter (Éd.), Durkheim et Weber. Vers la fin des malentendus?, L'Harmattan, 1994. 
l'élaboration d'une définition préalable se heurte à des difficultés évidentes: outre qu'elle aboutit nécessairement à un artefact dont il n'est pas certain qu'il corresponde à un phénomène empirique «réel», elle risque de conceptualiser des orientations théoriques spécifiques exclusives d'approches différentes et d'être alors incapable de remplir la tâche que nous lui assignons.

Il faut donc, dans un cas comme celui-ci, procéder de façon durkheimienne, c'est-à-dire partir de deux principes ${ }^{8}$. En premier lieu, il faut considérer que si les individus disent éprouver des émotions, il serait mal venu de penser qu'ils se trompent et, surtout, qu'ils ne savent pas ce qu'ils éprouvent. (On sait que le véritable «coup de force» de Durkheim dans Les Formes élémentaires de la vie religieuse est d'avoir pensé, dans une époque plutôt rationaliste et athée, que si les individus adoraient religieusement quelque chose, c'est que cette chose-là devait bien exister !) On considérera donc que les émotions sont des choses qui existent, puisqu'elles sont ressenties, et que les individus peuvent les identifier et les décrire parfaitement même s'ils ne sont pas nécessairement en mesure de les expliquer. (Durkheim se méfie toujours - c'est un euphémisme - des explications des acteurs ${ }^{9}$, mais jamais de la sincérité de leurs représentations: si quelqu'un cherche à m'expliquer pourquoi il est ému, je peux toujours le suspecter de se tromper; en revanche, s'il me déclare qu'il est ému, je n'ai pas le droit d'en douter (sauf s'il ment volontairement) pas plus que je ne peux critiquer a priori les descriptions qu'il fait de son état d'émotion.) En second lieu, selon la bonne vieille méthode proposée dans Les Règles, il faudra essayer de s'en tenir aux aspects objectifs (empiriques) du phénomène et limiter le contenu de la définition à ces aspects.

Bref, nous proposons de partir non pas d'un concept théorique mais d'un concept purement phénoménologique (on pourrait d'ailleurs discuter de la question de savoir s'il s'agit alors d'un concept...). Nous voudrions ainsi pouvoir retenir, dans le concept d'émotion, une définition qui soit à la fois la plus extérieure possible (c'est-à-dire qui ne fasse pas intervenir de savoir «théorique » explicite) et la moins litigieuse possible (c'est-à-dire qui fasse l'objet d'un relatif consensus). Toutefois, parmi toutes les dimensions de ce concept, nous privilégierons évidemment celle dont la pertinence sociologique est la plus évidente: la définition que nous rechercherons sera donc en rapport avec l'action - et, comme il n'y d'action empirique qu'individuelle, elle devra concerner l'action individuelle.

\section{Un essai de conceptualisation sociologique}

Dans le langage courant, le terme d'émotion renvoie à des phénomènes empiriquement bien réels puisqu'ils désignent une expérience que chaque individu est normalement en mesure de faire, et que cette expérience est sans grande ambiguïté. Chacun sait très bien ce qu'est une émotion: nous en avons tous et, même si nous ne savons pas les expliquer, nous sommes en mesure de décrire assez précisément ce que nous ressentons quand nous en éprouvons une. Nous possédons

Émile Durkheim, Les Règles de la méthode sociologique [1895], Paris, P.U.F., 1995.

Charles-Henry Cuin, Sociologie sans paroles: Durkheim et le discours des acteurs, in Massimo Borlandi et Mohamed Cherkaoui (Éd.), Le Suicide. Un siècle après Durkheim, Paris, P.U.F., 2000, p. $125-146$. 
même, pour les décrire, toute une série d'expressions qui, pour être métaphoriques, n'en sont pas moins parfaitement explicites et, surtout, sans ambiguiité: nous disons d'une émotion qu'elle nous «submerge», nous «envahit», nous «domine», nous « prend», nous « surprend», nous «porte», nous «affecte», nous «frappe», que nous sommes «sous le coup de l'émotion»-avec, bien sûr, une intensité variable: du simple trouble à la plus totale stupéfaction. Il existe donc bien une expérience commune de l'émotion.

Quelle est cette expérience? Elle est celle d'une sorte de désappropriation involontaire de nous-mêmes, qui peut nous conduire à faire ce que nous ne voulons pas faire ou à ne pas faire ce que nous voulons. Nous pouvons d'ailleurs identifier nos émotions, les nommer: la colère, l'angoisse, la répulsion, le chagrin, l'indignation, mais aussi la jouissance, le ravissement, l'extase, l'enthousiasme, etc. En outre, nous faisons fort bien la différence entre sentiment et émotion. Nous ne confondons pas la peur et l'angoisse, l'amour et l'excitation sexuelle, l'irritation et la colère, le plaisir et la jouissance, la tristesse et le chagrin; la crainte ou le déplaisir ne sont pas le dégoût ou la répulsion ${ }^{10}$. Nous ne concevons pas l'émotion simplement comme l'exacerbation d'un sentiment. Nous pensons que nous éprouvons des sentiments en permanence, alors que l'émotion est une expérience ponctuelle et - heureusement pour certaines et malheureusement pour d'autres - plus rare. D'ailleurs, quand on dit qu'on éprouve un sentiment, on n'a rien dit tant que l'on n'a pas précisé de quel sentiment il s'agit; en revanche, affirmer que l'on éprouve une émotion est suffisant pour qualifier une expérience et la communiquer à autrui.

Nous proposerions donc volontiers la définition suivante: l'émotion est une expérience psycho-physiologique que le sujet éprouve comme une altération plus ou moins importante de sa rationalité, voire de son intentionnalité - c'est-à-dire une diminution plus ou moins importante des contrôles conscients qu'il exerce (ou pense exercer) habituellement sur ses conduites. Cette diminution du contrôle affecte à la fois l' «esprit » et le corps. La partie consciente de l'esprit est envahie de sensations auxquelles le sujet a de plus ou moins grandes difficultés à résister. Dans le même temps, le corps peut éventuellement adopter des attitudes, des postures, des comportements qui échappent à la volonté du sujet. L'émotion peut évidemment varier en intensité, être plus ou moins forte, plus ou moins irrésistible. Dans sa forme la plus intense, l'individu n'est plus le sujet de ses conduites, il se sent «hors de lui », son activité n'est plus qu'un comportement. Sous une forme de moindre intensité, il parvient plus ou moins difficilement à diriger ses conduites comme il le ferait dans des circonstances «normales». (Ainsi, si je suis en colère, je peux gifler celui qui m'a insulté, mais je peux aussi casser des assiettes ou me frapper la tête contre un mur: dans le premier cas il me reste un peu de rationalité, dans le second c'est mon intentionnalité elle-même qui est atteinte.)

Cette définition de l'émotion peut sans doute être critiquée sur bien des aspects en regard du contrat que nous nous sommes imposé. On peut d'abord relever que cette définition comporte quelques notions non purement descriptives

10 Voir la récente livraison de la revue Critique, «Penser les émotions », LV (625-626), juin-juillet 1999. 
(rationalité, intentionnalité); ensuite qu'elle est formellement négative («altération du contrôle conscient »); enfin et surtout qu'elle peut paraître reposer sur une conception théorique - discutable, de surcroît - de l'action humaine. Il nous faut donc la défendre un peu.

Il n'est bien sûr absolument pas question de considérer que l'action humaine est, en général, intentionnelle et rationnelle (voulue et raisonnable), ou encore que l'intentionnalité et la rationalité constitueraient l'idéal et leur contraire une déviance. Dans la réalité, l'action humaine est au moins aussi souvent routinière ou affective que stratégique et délibérée, de même que ses objectifs sont assez souvent obscurs, voire inexistants. Nous ne disons pas non plus que les individus désirent toujours agir avec une parfaite lucidité et une parfaite rationalité: si c'était le cas, ils ne se complairaient pas aussi souvent dans l'émotion, et le marché des émotions ne serait pas aussi florissant (par exemple, dans le sport, les arts ou autres divertissements $)^{11}$. Nous disons simplement que les individus décrivent des expériences dans lesquelles il leur semble pouvoir constater qu'un éventuel «contrôle d'eux-mêmes » leur est difficile ou même impossible.

\section{Une substruction de la typologie weberienne de l'action ${ }^{12}$}

La typologie weberienne de l'action peut être en partie reconstruite à partir des deux dimensions centrales de notre définition des émotions : l'intentionnalité et la rationalité. Ces termes sont des concepts sociologiques parfaitement classiques. L'intentionnalité, c'est la capacité de l'acteur de donner des buts à son action en fonction de ses préférences. (En fait, Weber emploie le terme de «signification»: une action, au sens fort du terme, est nécessairement significative, douée de sens, pour son acteur.) La rationalité, c'est tout simplement la capacité de choisir les moyens d'atteindre ces buts. (Elle peut être «objective», et l'on déclarera rationnelle l'action qui choisit des moyens techniquement appropriés aux fins, ou «subjective» lorsque l'acteur estime que son choix est techniquement adapté. La rationalité étant toujours «limitée » ${ }^{13}$, c'est la rationalité subjective qui, évidemment, intéresse le sociologue.)

Une action, dans le langage de Weber, est toujours intentionnelle puisqu'elle consiste à employer des moyens pour atteindre des fins. Une conduite non intentionnelle (en fait: un simple comportement) ne peut donc pas, par définition, être rationnelle - du moins au sens «subjectif ». En revanche, une action intentionnelle peut être rationnelle ou non-rationnelle: elle est rationnelle si les moyens utilisés ont été délibérés par l'acteur en fonction de leur adéquation aux buts, elle est nonrationnelle dans le cas contraire.

Le tableau suivant permet d'y voir plus clair:

\footnotetext{
11 Norbert Elias et Eric Dunning, Sport et civilisation. La violence maîtrisée [1986], Paris, Fayard, 1994.

12 Sur l'opération typologique de substruction, voir Allen Barton, Le concept d'espace d'attributs en sociologie, in Raymond Boudon et Paul Lazarsfeld (Éd.), Le Vocabulaire des sciences sociales, Paris-La Haye, Mouton, 1967, p. 148-170.

13 James March et Herbert Simon, Les Organisations [1958], Paris, Dunod, 1974.
} 


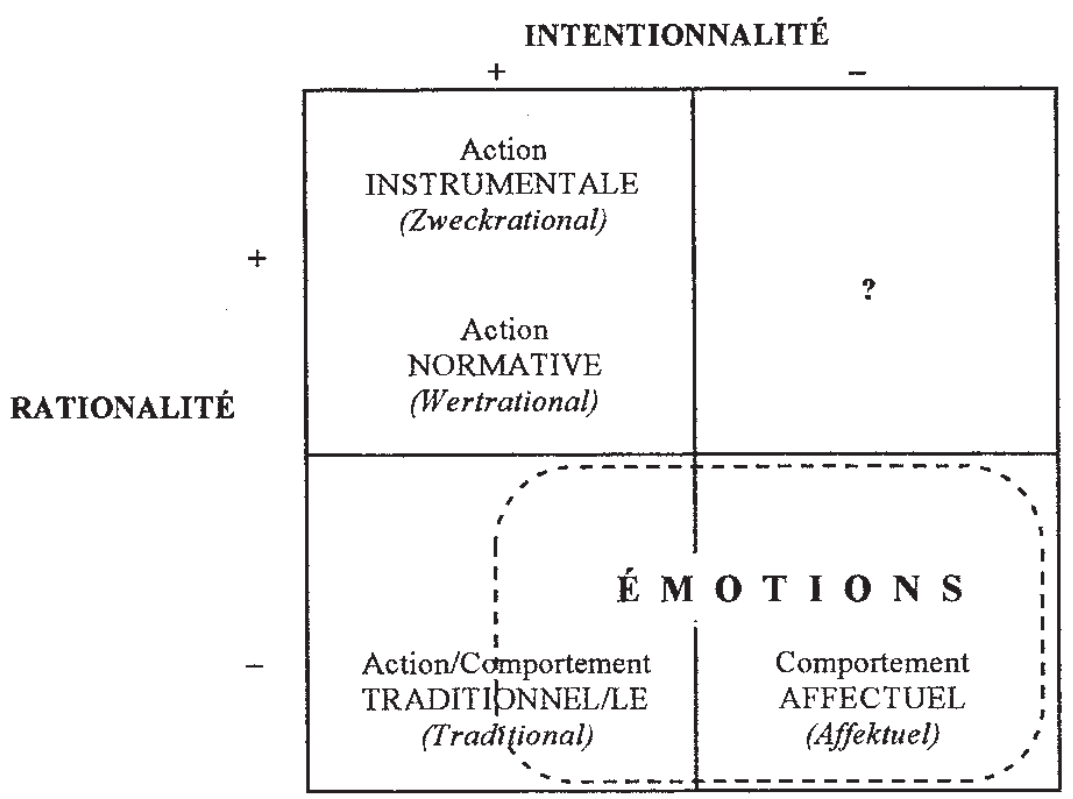

\section{Commentaire du tableau:}

Rappelons que, parmi les conduites intentionnelles, certaines sont rationnelles et d'autres non. Selon Weber, les actions rationnelles peuvent l'être

- par rapport aux buts (Zweckrational: Action «instrumentale» ou «rationnelle en finalité») lorsque les moyens sont choisis en fonction de leur efficacité à atteindre les buts,

- ou par rapport aux valeurs (Wertrational: Action «normative») lorsque les moyens sont choisis en fonction de leur valeur intrinsèque - que cette valeur soit morale, esthétique ou encore religieuse.

Il s'agit évidemment de types «idéaux» qui ne se rencontrent guère en l'état dans la réalité. Ils ne sont en effet ni exclusifs, ni les seuls possibles. Chez les gens qui ne sont ni des saints ou des héros, ni des cyniques et des salauds (c'est-à-dire chez beaucoup d'entre nous), l'action est orientée à la fois en fonction des buts et en fonction des valeurs: si je veux m'enrichir et si j'ai le choix entre travailler et voler, à efficacité égale, je choisirai de travailler.

Dans le cas des actions non-rationnelles, le choix des moyens n'est pas délibéré. Il est plutôt comme «imposé» par l'acteur à lui-même. Weber en distingue deux types:

- Les actions «traditionnelles» (Traditional). C'est le type le plus ambigu: ces actions sont conduites soit au nom d'une coutume, d'une tradition, d'une façon de faire qui n'a jamais variée (et l'on est alors très proche de l'action «normative»), soit de façon «routinière », c'est-à-dire en fonction d'une habitude invétérée (on est donc ici à la limite entre intentionnalité et non intentionnalité: l'individu qui agit de façon routinière obéit plus à des «pulsions » qu'à sa propre volonté). ${ }^{14}$

14 «Le comportement strictement traditionnel [...] se situe absolument à la limite, et souvent audelà, de ce qu'on peut appeler en général une activité orientée 'significativement'. Il n'est, en effet, très souvent qu'une manière morne de réagir à des excitations habituelles, qui s'obstine dans la direction d'une attitude acquise autrefois. La masse de toutes les activités quotidiennes familières se rapproche de ce type [...]» (Max Weber, Économie et société/1 [1921], Paris, PlonPresses Pocket, 1995, p. 55) Voir également: Ibid., p. 29. 
- Les actions «affectuelles» (Affektuel), en revanche, caractérisent sans ambiguïté celles qui sont conduites sous l'effet de sentiments forts ou d'émotions. On est là dans le domaine exemplaire de l'émotion. Weber est d'ailleurs très clair sur ce point: «Agit de manière affectuelle celui qui cherche à satisfaire le besoin d'une vengeance actuelle, d'une jouissance actuelle, d'une félicité contemplative actuelle ou encore celui qui cherche à se débarrasser d'une excitation actuelle (peu importe s'il le fait d'une manière indigne ou sublime)» ${ }^{15}$.

On se trouve donc clairement dans la colonne de droite du tableau : celle des conduites non intentionnelles. Par définition, elles sont aussi non rationnelles.

Reste le cas de la dernière case. Dans la typologie weberienne, elle n'existe pas, puisque toute conduite non intentionnelle est - ipso facto - non rationnelle. (A fortiori, elle ne peut pas être «sociale» puisqu'elle n'est pas orientée en fonction de l'action d'autrui.) Pourtant, on montrera plus bas que, chez Durkheim, ce cas semble bel et bien exister: il serait même, pour cet auteur, particulièrement représentatif de l'action sociale dans ce qu'elle a de plus essentiel.

\section{II - WEBER: LES RESSORTS ÉMOTIONNELS DE LA RATIONALISATION}

On voudrait montrer ici que la sociologie weberienne repose tout entière sur le constat du caractère non rationnel, voire non intentionnel, de très larges pans de l'action humaine - et, en particulier, de son caractère émotionnel. Ce constat ne fonde pas seulement les analyses classiques du rôle du «charisme» ou du phénomène religieux dans la vie sociale et dans l'évolution des sociétés. Il est aussi à l'origine de deux redoutables questions : la première, épistémologique, est celle de la capacité d'une méthode foncièrement individualiste à expliquer des conduites dont beaucoup sont étrangères à la raison ou à la volonté; la seconde, théorique, consiste dans la conciliation du caractère souvent routinier ou affectif de ces conduites avec une dynamique sociale marquée par la «rationalisation». Ce sont ces gageures qui conduisent Weber à élaborer, dans un cas, une méthodologie complexe de la «compréhension sociologique», dans l'autre, à développer une théorie originale des ressorts du charisme et de sa «routinisation».

\section{Comprendre les conduites non rationnelles}

La sociologie weberienne repose, on le sait, sur un paradigme individualiste. Les phénomènes macrosociaux (i.e., les institutions sociales) sont conçus comme résultants de l'agrégation de conduites individuelles, c'est-à-dire d'actions concrètes d'individus concrets. Leur explication requiert donc celle de ces conduites et, par effet, leur «compréhension» - c'est-à-dire la saisie de leur signification, de leur sens. Ce sens est constitué par les «motifs» qui ont poussé l'acteur à adopter une conduite particulière.

Toutefois, Weber distingue soigneusement la compréhension «actuelle» [aktuelles Verstehen] de la compréhension «explicative» [erklärendes Verstehen $].{ }^{16}$ La première relève de l'évidence de ce sens pour un observateur: les motifs de l'acteur sont immédiatement accessibles et il ne fait pas de doute que la conduite observée est le résultat de l'actualisation de ces motifs. La seconde

$\begin{array}{ll}15 & \text { Ibid., p. } 56 . \\ 16 & \text { Ibid., p. } 34 .\end{array}$ 
démarche vise à imputer l'action à des causes. Dans ce cas, la saisie du sens d'une conduite doit permettre de l'expliquer, c'est-à-dire de connaître les causes qui ont conduit l'acteur à faire ce qu'il fait. Pour cela, il faut accéder aux motifs.

Dans certains cas, la compréhension «actuelle» permet l'explication. Par exemple, lorsque j'observe un commerçant faisant des additions ou un bucheron maniant sa hache (sic), je comprends immédiatement pourquoi ils font ce qu'ils font, et je peux donc l'expliquer: l'un et l'autre font leur métier. Mais dans beaucoup d'autre domaines (la plupart d'entre eux, selon Weber), cette compréhension «actuelle» ne conduit pas à l'explication, ou n'y conduit que difficilement. Ainsi, pour reprendre un exemple fameux de Jaspers, quand j'observe une mère giflant son enfant, je comprend bien le sens de cette conduite: la mère est en colère, et la colère peut favoriser ce genre de conduite «émotionnelle». Pourtant les raisons de cette émotion - et, donc, de cette conduite - me restent inconnues. Je peux évidemment faire des hypothèses: l'enfant n'a pas été sage, la mère est dépressive, elle est d'un tempérament colérique ou violent, etc. En fait, il n'y a qu'un seul cas où je pourrais expliquer cette gifle par compréhension «actuelle»: c'est celui où il n'y aurait pas d'émotions en jeu, c'est-à-dire si l'action de la mère était purement rationnelle en finalité, «instrumentale » (la mère a giflé l'enfant afin qu'il cesse de se conduire comme il le fait et/ou qu'il apprenne à agir différemment); mais, bien évidemment, je ne peux pas être sûr que ce soit le cas.

En fait, c'est là la vraie raison de la construction par Weber de ce type-idéal de la rationalité «instrumentale». Il ne sert pas à décrire des conduites; il sert essentiellement de modèle de référence à l'analyse de l'action. L'action rationnelle en finalité est la seule qui soit à la fois et en même temps intégralement compréhensible et intégralement explicable. Aucun autre type d'action ne l'est aussi parfaitement. La méthode weberienne consiste donc, pour expliquer une conduite réelle, à considérer celle-ci comme une déviation d'une conduite rationnelle en finalité. Et c'est la distance existant entre cette conduite idéal-typique et la conduite réelle qui permet de faire des hypothèses sur les motifs de l'acteur réel. Si la mère qui a giflé l'enfant avait agi rationnellement en finalité, elle ne l'aurait pas fait, par exemple, s'il pleurait déjà (ou s'il était parfaitement sage), ou en étant rouge de colère. Or, l'enfant pleurait déjà et la mère était rouge de colère: les motifs de la mère sont donc essentiellement émotionnels.

Dans l'Éthique protestante, Weber ne procède pas autrement. Je peux aujourd'hui - expliquer par «compréhension actuelle» que l'entrepreneur capitaliste cherche à faire du profit et en réinvestisse une certaine partie, car il s'agit là d'une action parfaitement rationnelle en finalité. En revanche, il m'est plus difficile de comprendre d' emblée pourquoi le calviniste du XVI ${ }^{\mathrm{e}} \mathrm{s}$. travaille aussi durement et ne profite pas du fruit de son travail. Soit il est fou, soit il est pervers. Si je fais plutôt l'hypothèse qu'il est rationnel, il doit alors avoir de bonnes raisons de faire ce qu'il fait. Mais, à l'évidence, ces raisons ne sont pas de type utilitaire. Il ne reste plus que des raisons de type moral. Et, de fait, en examinant son éthique religieuse, ces bonnes raisons apparaissent assez nettement: elles ne sont pas de type «instrumental» mais de type «normatif».

Ainsi, c'est la prise en compte du caractère émotionnel (i.e., non rationnel, voire non intentionnel) d'un très large champ de l'action humaine qui conduit Weber, non seulement à préconiser la méthode idéal-typique, mais aussi et surtout à affirmer le caractère indissociable des démarches «compréhensive» et «expli- 
cative $»^{17}$. En fait, si l'analyse sociologique requiert la «compréhension » du sens des conduites individuelles, la «méthode compréhensive» ne saurait se limiter à la saisie de la subjectivité consciente des acteurs. Weber le souligne d'ailleurs avec force: «Dans la grande masse des cas, l'activité réelle se déroule dans une obscure semi-conscience ou dans la non-conscience du 'sens visé'. L'agent le 'sent' imprécisément plus qu'il ne le connaît ou ne le 'pense clairement'; il agit dans la plupart des cas en obéissant à une impulsion où à la coutume. Ce n'est qu'occasionnellement qu'on prend conscience du sens (qu'il soit rationnel ou irrationnel) de l'activité, et dans les cas de l'activité similaire d'une masse c'est souvent le fait de quelques individus seulement.» ${ }^{18}$

\section{Les paradoxes du processus de rationalisation}

Ce que l'on retient le plus - et le mieux - de Max Weber c'est qu'il est le sociologue de la rationalisation. La rationalisation, c'est cette tendance apparemment irrépressible des sociétés occidentales à introduire toujours plus de rationalité dans tous les domaines de l'activité et de la vie sociale. Cette tendance se manifeste aussi bien dans le domaine du politique (avec la généralisation de l'autorité «rationnelle-légale »), de l'administration (avec le triomphe de la bureaucratie) ou de l'économie (avec l'invention du capitalisme) que dans celui du droit, de la religion $^{19}$ (en particulier dans le cas du protestantisme), ou même encore de l'art (en particulier dans le domaine de la musique ${ }^{20}$ ). Si l'on peut parler de rationalisation et de rationalité, c'est bien parce que, dans chacun de ces domaines, les pratiques sociales deviennent plus réflexives, plus organisées, plus efficaces; parce qu'elles se fondent moins sur la spontanéité et plus sur la règle, moins sur le hasard et plus sur la prévision. Les pratiques sont moins des comportements et plus des conduites: elles poursuivent des buts qui ont une signification pour les individus, et elles cherchent à atteindre ces buts en utilisant des moyens qui sont choisis en fonction de leur adaptation à ces buts. Bref, les activités deviennent à la fois plus intentionnelles et plus rationnelles. Et cette rationalisation constitue, selon l'expression de Weber, «le destin de notre temps».

On pourrait donc croire que, dans ce processus, les émotions jouent un rôle entièrement négatif, que c'est essentiellement contre les émotions (ou, plus largement, contre l'affectivité) que ce processus a pu se développer et se mettre en place. S'il y a bien un système organisationnel ou un système économique qui excluent sans pitié toute émotion, c'est bien la bureaucratie ou le capitalisme!

Deux questions se posent donc. D'une part, comment des comportements non rationnels ou peu rationnels, souvent purement émotionnels, peuvent-ils conduire à l'émergence d'institutions caractérisées par une rationalité toujours plus grande? D'autre part, comment, dans un monde caractérisé par la rationalité, les comportements individuels peuvent-ils conserver une large part de routine ou d'affectivité?

\footnotetext{
17 «Nous appelons sociologie [...]une science qui se propose de comprendre par interprétation l'activité sociale et par là d'expliquer causalement son déroulement et ses effets », ibid., p. 28.

18 Ibid., p. 51.

19 Max Weber, Sociologie religieuse, Paris, Gallimard, 1996.

20 Max Weber, Sociologie de la musique [1921], Paris, Métailié, 1998.
} 


\section{De l'action émotionnelle à l'action rationnelle}

La première question est au centre de la très célèbre analyse présentée dans L'Éthique protestante et l' esprit du capitalisme de 1904-0521. Il n'est pas question de reprendre ici l'intégralité de la démonstration weberienne ${ }^{22}$. On se contentera de formuler deux observations.

D'abord, si l'on en croit Weber, c'est une véritable émotion qui est à l'origine de la conduite sociale du calviniste. Cette émotion est l'angoisse. Pour des raisons religieuses, c'est-à-dire qui tiennent à une certaine représentation du rapport entre Dieu et les hommes, le calviniste est littéralement effrayé de ne pas savoir quel sera son sort dans l'Au-delà, et de ne rien pouvoir faire pour gagner la compassion de Dieu. Son Dieu, en effet n'est pas un dieu compatissant, c'est un dieu terrible qui a déjà choisi ses Élus sans que ces derniers le sachent. Mais il y a quand même un moyen de reconnaître ces Élus: l'Élu de Dieu est nécessairement un individu qui a parfaitement accompli son destin sur terre - destin qui est de travailler avec succès, c'est-à-dire d'avoir réussi sa vie professionnelle. La seule façon de calmer cette angoisse, c'est donc de vérifier sans relâche si l'on fait partie des Élus et, donc, de travailler sans répit et avec le plus de chances possibles de succès - c'està-dire le plus rationnellement possible.

On connaît la suite. Étant donné que le calviniste est également tenu à un mode de vie ascétique, il va travailler avec ardeur et méthode mais sans jouir du fruit de son travail, ce qui va le conduire à capitaliser de l'argent qu'il ne pourra que réinvestir pour ne pas le dépenser. Et c'est cette morale (et la conduite qu'elle inspire) qui, selon Weber, aurait constitué un puissant facteur de développement et de légitimation du capitalisme comme système économique.

La question n'est pas de discuter cette thèse, qui est d'ailleurs fort discutable ${ }^{23}$, mais de souligner le rôle joué par une émotion - l'angoisse de l'élection divine (la certitudo salutis) - non seulement dans l'apparition d'un type d'action sociale mais aussi dans le développement d'une institution économique. Dans ce processus, cette émotion ne constitue sans doute pas un point de départ, mais plutôt une variable intermédiaire nécessaire. Le véritable point de départ (dans la perpective weberienne, s'entend), c'est l'apparition historique d'un nouveau système de valeurs religieuses dans l'Europe du XVI ${ }^{\mathrm{e}}$ siècle: le protestantisme calviniste. Le plus étonnant, c'est d'ailleurs que cette morale religieuse est profondément séculière et ascétique. Elle combat toute forme d'irrationalité et, pour ce faire, invite le croyant à réprimer ses émotions le plus sévèrement possible. (Certaines sectes protestantes de type «méthodiste» ont d'ailleurs des positions particulièrement radicales dans ce domaine ${ }^{24}$.) En fait, et comme par un effet pervers, c'est cette répression de l'irrationnel qui donne naissance à l'angoisse: si l'on ne peut pas communiquer avec Dieu, si l'on ne peut pas «négocier» avec lui, alors l'existence du croyant devient effrayante - et le seul moyen de calmer cette angoisse est de rationaliser ses propres conduites. Dans ce cas, cette rationalisation prend une

\footnotetext{
21 Max Weber, L'Éthique protestante et l' esprit du capitalisme [1904-05], Paris, Plon, 1964.

22 Voir, entre autres: Annette Disselkamp, L'Éthique protestante de Max Weber, Paris, P.U.F., 1994.

23 Philippe Besnard, Protestantisme et capitalisme. La controverse post-weberienne, Paris, A. Colin, 1970 .

24 Max Weber, L'Éthique protestante..., op. cit., p. 181-186.
} 
forme bien particulière: celle de la rationalité axiologique (i.e., normative). Cela signifie que ce qui va guider l'action du calviniste, ce ne sera pas d'atteindre des buts sociaux (la richesse, le pouvoir, le prestige) mais de réaliser des buts moraux, c'est-à-dire de se conduire comme s'il était un élu de Dieu. On vient donc de passer de l'émotion à la raison, plus précisément du «comportement affectuel» de la typologie weberienne à l' «action normative» (ou «rationnelle par rapport aux valeurs»).

\section{Des conduites rationnelles aux institutions rationnelles}

Poursuivons la démonstration de Weber. Ce processus va connaître un sort bien étrange, tout à fait paradoxal et, d'une certaine manière, tragique. En effet, ce type d'action va se routiniser (selon l'expression weberienne consacrée). Cela signifie que les conduites vont demeurer les mêmes, perdurer, mais que leurs raisons d'être vont changer. Que l'on va continuer à amasser (ou à tenter d'amasser) rationnellement la plus grande richesse possible non plus au nom de valeurs morales mais tout simplement par obligation, parce que l'on ne peut plus faire autrement. La raison ne commandera plus d'agir ainsi parce que c'est moral, mais parce que c'est utile et nécessaire. On va passer de la «rationalité normative» à la rationalité «instrumentale» (ou utilitaire, purement égoïste, sans justification morale).

Dans la perspective weberienne, l'explication de ce bouleversement est toute simple. Il s'est passé, en effet, que ces conduites morales ont débouché sur la victoire du capitalisme (c'est-à-dire que, si elles n'ont pas directement produit le capitalisme, elles ont été un «puissant levier» (sic) de sa victoire). Désormais, non seulement le capitalisme n'a plus besoin de ce moteur moral et de cette légitimité morale, mais aussi et surtout il va constituer le nouvel espace socio-économique des sociétés occidentales - Weber parle d'une nouvelle «cage d'acier»: après le règne des «idées », on passe au règne des «intérêts ». C'est donc ainsi que le capitalisme, sinon issu de, du moins favorisé et légitimé dans son émergence par l'agrégation d'un certain type de conduites individuelles, va à son tour engendrer de nouvelles conduites individuelles. Il se trouve qu'ici ce sont les mêmes conduites (amasser rationnellement un profit maximum et le réinvestir) mais pour des buts différents - non pas par souci moral mais par souci bien pratique: parce que, si on ne réinvestit pas une part de plus en plus importante du profit, on risque fort de perdre tout son capital. C'est tout le sens de la fameuse phrase: «Le puritain voulait être un homme besogneux - et nous sommes forcés de l'être.» ${ }^{25}$

Peut-être même pourrait-on aller jusqu'à dire que l'angoisse qui poussait à l'action normative va changer de forme: elle n'est plus l'angoisse de l'élection divine mais l'angoisse de l'échec social, voire de la ruine. Et, à son tour, cette nouvelle forme d'angoisse va pousser à une nouvelle forme de rationalité: celle de l'action instrumentale (ou rationnelle par rapport aux buts). Une telle interprétation n'est d'ailleurs pas absente du texte de Weber ${ }^{26}$.

\footnotetext{
25 Ibid., p. 249.

26 Ibid., p. 250 sq.
} 


\section{Des émotions à la rationalisation: le rôle du charisme}

Mais il reste une dernière question: comment, de façon générale, les sociétés passent-elles de la tradition, avec ses routines largement irrationnelles, à la modernité rationalisée? Comment la rationalité pénètre-t-elle ce qui est son repoussoir permanent? Comment peut-elle naître de quelque chose qui l'ignore et qui la nie ? $^{27}$

Weber, à ce propos, évoque ce qu'il appelle le «charisme» de certains individus, de certaines doctrines. Si l'on prend la notion de charisme dans son sens le plus juste, il équivaut à l'émotion profonde que ces individus et ces doctrines provoquent parmi les masses. C'est le cas des prophètes, des devins, des grands mystiques, des grands chefs. Ces individus «charismatiques» ne sont évidemment ni désintéressés ni dupes de leur pouvoir. Au contraire, ils utilisent en général leur charisme de façon tout à fait rationnelle pour atteindre leurs buts propres, et ils sont d'autant plus efficaces qu'ils fondent leur discours révolutionnaire sur des sentiments et des représentations très profondément ancrés chez ceux qui les écoutent ${ }^{28}$. C'est de cette façon qu'ils provoquent ces émotions collectives d'enthousiasme, voire d'extase. Et ce sont ces émotions qui permettent aux masses de transgresser les normes routinières de la tradition, parfois de transformer le monde.

Ainsi, nous dit Weber, le processus normal de l'évolution de l'autorité charismatique est de se «routiniser»-c'est-à-dire soit de reconstituer un nouvel ordre traditionnel soit, au contraire, de passer de la tradition à un ordre social plus rationalisé. Si cette «routinisation du charisme» conduit souvent à un degré supérieur de rationalité, c'est parce que, pour pouvoir se survivre à lui-même, le nouvel ordre social doit s'adapter à la réalité, s'institutionnaliser, renforcer par des règles ce qui ne tenait qu'à des sentiments. ${ }^{29}$

Si l'on fait nos comptes, c'est bien à trois moments du processus de rationalisation décrit par Max Weber que l'on retrouve les émotions. D'abord, dans le succès de l'irruption du charisme dans la tradition: un prophète, un chef suscite l'enthousiasme, l'extase des foules (Weber qualifie ces phénomènes d' «orgiaques »). Ensuite, dans la traduction de ces nouvelles valeurs en émotions qui vont susciter des conduites nouvelles: dans le cas particulier du protestantisme calviniste, ces valeurs engendrent une angoisse qui, à son tour, va engendrer un certain type de comportement économique. Enfin, dans le passage de la rationalité axiologique à la rationalité instrumentale, où le moteur émotionnel des conduites va changer de nature: les émotions ne sont plus religieuses (par ex., l'angoisse de l'élection divine) mais totalement séculières (par ex., l'avidité d'un profit sans limites).

Il apparaît donc clairement que, pour Weber, c'est l'irrationalité même de certaines conduites collectives qui constitue l'un des moteurs essentiels du changement social. Tout se passe comme si la rationalisation de l'action traditionnelle n'était rendue possible que par la médiation d'une certaine irrationalité des

27 Voir: Philippe Reynaud, Max Weber et les dilemmes de la raison moderne [1987], Paris, P.U.F., 1996.

28 Michael Walzer, Critique et sens commun. Essai sur la critique sociale et ses interprétations, La Découverte, 1990.

29 Max Weber, Économie et sociétél1, op. cit., p. 326 sq. 
conduites - comme si, ainsi que le souligne Hans Joas, la créativité devait nécessairement passer par l'irrationnel, par des émotions ${ }^{30}$.

C'est pour cette raison que Max Weber est si pessimiste pour l'avenir de nos sociétés hyper-rationalisées. Selon lui, les émotions y sont si fortement refoulées qu'il voit mal comment de nouveaux charismes pourraient s'y développer. Il exprime cette crainte dans une formule terrible à travers laquelle il croit pouvoir dépeindre les «derniers hommes » de notre civilisation: «Spécialistes sans vision et voluptueux sans cœur - ce néant s'imagine avoir gravi un degré de l'humanité jamais atteint jusque là.» ${ }^{31}$

Malheureusement, on le sait, Weber se trompait. S'il avait raison d'être pessimiste, il se trompait de pessimisme: ce n'était pas la fin des charismes que l'Europe allait connaître quelques années plus tard mais, au contraire, l'un des plus monstrueux charismes de l'Histoire. Et s'il se trompait, c'est parce qu'il n'avait pas compris que cette rationalisation poussée à l'extrême - ce «désenchantement du monde - allait provoquer une réaction de dégoût (et le dégoût est bien une émotion!), et que cette émotion allait être soigneusement récupérée par un prophète fou $^{32}$. Bref, il se trompait parce qu'il n'avait pas appliqué à son propre siècle la théorie qu'il avait si brillamment appliquée à l'Histoire de l'humanité. Sur ce point, la pensée de Durkheim est sans doute moins pessimiste, et peut-être plus pertinente.

\section{III - DURKHEIM: LES DILEMMES SOCIAUX DE LA VIE ÉMOTIONNELLE}

La position de Durkheim vis-à-vis des émotions est complexe. En fait elle est plus ambivalente qu'ambiguë, car elle concerne deux conceptions du rôle des émotions dans la vie sociale. Ces deux conceptions, très différentes l'une de l'autre, voire opposées, ont chacune leur cohérence propre. Le problème, c'est qu'elles se succèdent au cours de l'évolution de la pensée durkheimienne, et souvent se superposent. On tentera toutefois de montrer qu'elles ne sont pas contradictoires mais sont au contraire complémentaires si l'on conceptualise un peu cette notion.

\section{L'évolution du statut des émotions}

\section{Phase 1: La Régulation contre les émotions}

Chez celui que j'appellerai le premier Durkheim (grosso modo celui de Bordeaux, jusqu'au tournant des années 1900), les émotions ont un statut très clair: elles relèvent, selon la dichotomie classique, du domaine de la «nature » par opposition à celui de la «culture» ou encore de la «socialisation».

Durkheim, comme tous ses contemporains, est évolutionniste. Dans le domaine qui nous intéresse, il estime que l'évolution culturelle est analogue à

\footnotetext{
30 Hans Joas, La Créativité de l'agir [1992], Paris, Cerf, 1999.

31 Max Weber, L'Éthique protestante et l' esprit du capitalisme, op. cit., p. 251.

32 Ian Kershaw, Hitler. Essai sur le charisme en politique, Paris, Gallimard, 1995.
} 
celle du psychisme individuel. Le psychisme du «sauvage» et celui du petit enfant sont donc analogues. Tous deux sont des êtres continuellement agités par des émotions aussi violentes que passagères et qui les font se comporter de manière à la fois imprévisible, passionnelle et, donc, totalement a-sociale - « amorale » dit Durkheim. Il suffit de relire le cours sur L'Éducation morale donné à partir de 1889:

«L'enfant passe d'une impression à l'autre, d'une occupation à l'autre, d'un sentiment à l'autre, avec la plus extraordinaire rapidité. Son humeur n'a rien de fixe; la colère y naît et s'y apaise avec la même instantanéité; les larmes succèdent aux rires, la sympathie à la haine, ou réciproquement, sans raison objective ou, tout au plus, sous l'influence de la plus légère circonstance.» ${ }^{33} \mathrm{Et}$, quelques lignes plus loin: «Sur ce point [...] comme sur bien d'autres, l'enfant ne fait que reproduire un des caractères distinctifs de l'humanité primitive. Les peuples qui n'ont pas encore dépassé les formes les plus inférieures de la civilisation se font, en effet, remarquer par cette même mobilité d'idées et de sentiments, la même absence de suite dans la conduite individuelle. L'incident le plus insignifiant suffit pour opérer, dans la conscience de l'adulte, les plus surprenantes révolutions. Un geste heureux, une grimace, un mot, et la colère la plus farouche se transforme en sentiments bienveillants; ou bien, au contraire, des menaces de mort succèdent aux plus chaudes démonstrations d'amitié.» ${ }^{34}$

On n'a donc aucune peine à retrouver des ces propos la définition des émotions que nous proposions plus haut: des états psychiques apparemment désordonnés produisant des conduites apparemment non rationnelles et, tout aussi apparemment, non intentionnelles. Durkheim est d'ailleurs formel: il s'agit là, écrit-il, d' «une aliénation passagère de la personnalité » ${ }^{35}$. Pour caractériser ces états mentaux et les conduites qui leur correspondent, son maître mot est celui d' «irrégularité ». Est «irrégulier» ce qui se produit à la fois de manière intempestive (c'est-à-dire sans qu'on puisse l'attendre, le prévoir) et, surtout, de manière immesurée (c'est-à-dire exagérée par rapport aux causes qui le déclenchent et par rapport aux conséquences que l'on en attend). C'est donc ce qui ne correspond pas à une règle, à une norme - que cette norme soit une simple régularité empirique ou, plus concrètement, une loi: objective (qui s'impose naturellement) ou subjective (que l'on s'impose volontairement).

Si les conduites des enfants et des «primitifs » sont «irrégulières », c'est donc qu'elles correspondent à des états mentaux qui sont eux-mêmes irréguliers, non régulés. Or, Durkheim est un positiviste. Toute chose obéit à une règle: à une règle naturelle si c'est une chose naturelle, à une règle sociale si c'est une chose sociale. Pourtant les enfants et les «primitifs » semblent bien échapper à cette règle universelle de la régulation! Leurs conduites ne semblent être réglées ni par la biologie (de l'intérieur) ni par la culture (de l'extérieur). Dans Le Suicide, cette espèce de monstruosité est parfaitement soulignée: de tous les animaux, les êtres humains sont les seuls dont les conduites ne sont pas naturellement réglées. Plus précisément, à l'état de nature, les désirs humains sont tout à la fois sans buts pré-

\footnotetext{
33 Émile Durkheim, L'Éducation morale [1925], Paris, P.U.F., 1963, p. 110.

34 Ibid., p. 111.

35 Ibid., p. 112.
} 
cis et sans limites d'intensité. Ils sont purement pulsionnels mais ces pulsions ne sont pas fonctionnelles comme le sont les instincts animaux; elles sont une espèce d'énergie improductive dont la seule logique semble bien être de se libérer indéfiniment et sans raison. Or, par définition, des désirs infinis sont insatiables. Et si rien ne peut les satisfaire c'est moins à cause de leur puissance, de leur avidité, que parce que cette avidité est universelle. «Vouloir tout, c'est ne pouvoir être satisfait par rien.» ${ }^{36}$

C'est bien ce que l'on observe, souligne Durkheim, lorsque le système normatif d'une société est affaibli, lorsque la culture (ce qu'il appelle la «conscience collective») n'est plus en mesure de prescrire ou, du moins, de suggérer ce qu'il convient de désirer - c'est-à-dire à la fois les buts et les procédures de l'action. De telles situations d' «anomie » ravalent les individus au rang de jouets de leurs émotions. Ils sont en effet soit dans la boulimie (l'«infini du désir») soit au contraire dans l'anorexie, dans l'apathie. Ils sont dominés par deux émotions apparemment contraires - l'exaltation ou le désespoir - mais dont Durkheim montre bien qu'elles ont une origine commune - l'absence de régulation sociale - et que l'exaltation conduit au désespoir puisque, par définition, des désirs insatiables ne peuvent pas être satisfaits. Et toute l'explication durkheimienne du «suicide anomique » consistera à montrer que, dans de telles situations, les individus ont - pour parler comme R. Boudon - de «bonnes raisons» objectives de mettre fin à des expériences aussi pénibles ${ }^{37}$.

Tout ceci montre bien que, pour le premier Durkheim, la société se construit contre les émotions. Plus exactement, la vie sociale n'est possible que dans la mesure où les émotions primaires de l'individu sont tout à la fois contrôlées et socialisées. Le premier but de toute «éducation morale » est d'apprendre à l'enfant à maîtriser ses émotions en leur donnant un rôle fonctionnel, c'est-à-dire en en faisant des principes d'action. C'est pour cette raison que toute action pédagogique revêt d'abord une dimension régulatrice: l'apprentissage de ce que Durkheim appelle «l'esprit de discipline». Par « discipline» il faut entendre un apprentissage de règles qui doit permettre, d'une part, de régulariser les conduites en créant des habitudes et, d'autre part, d'obéir à une autorité ${ }^{38}$. En fin de compte, il s'agit ici de substituer au chaos d'une activité purement émotionnelle une orientation vers une activité soumise à une contrainte extérieure. Dans le langage weberien, il s'agit de passer de l'action «émotionnelle» à l'action «traditionnelle».

Il est cependant essentiel de souligner que le cours sur L'Éducation morale se situe à une période intermédiaire de l'œuvre durkheimienne. Durkheim a toujours insisté sur la complémentarité des deux dimensions de l'ordre social: la régulation et l'intégration. La première consiste dans la nécessité d'un corps de règles sociales, d'un «système normatif»; la seconde dans la nécessité d'une «conscience collective» forte et partagée. La première agit sur les individus en quelque sorte de l'extérieur, la seconde de l'intérieur.

\footnotetext{
36 Émile Durkheim, Le Suicide. Étude sociologique [1897], Paris, P.U.F., 1973.

37 Charles-Henry Cuin, Une méthode peut en cacher une autre: des 'Règles' au 'Suicide', in Ch-H. Cuin (Éd.), Durkheim d' un siècle à l' autre. Lectures actuelles des 'Règles de la méthode sociologique', Paris, P.U.F., 1997, p. 169-188.

38 Émile Durkheim, L'Éducation morale, Op. cit.
} 
Durant la première période de la réflexion de Durkheim (jusqu'au Suicide de 1897), c'est la problématique de la régulation qui est dominante. Le concept d'anomie occupe alors, dans son œuvre, une place centrale qu'il abandonnera par la suite, de façon très significative ${ }^{39}$. L'anomie est bien cet état de non-régulation ou de dérégulation de la société ou de certains segments sociaux. Ses effets sont décrits par Durkheim comme engendrant des conduites collectives de type essentiellement émotionnel. C'est le cas dans La division du travail social (1893) où il montre que l'affaiblissement des régulations économiques entraîne un état chronique de conflits qu'il désigne du terme d' «effervescence collective». Cette effervescence correspond à un état émotionnel puissant: les individus sont agités par des sentiments de frustration, d'égoïsme, d'agressivité non contenus et portés à leur paroxysme. Ce qui prévaut alors, c'est un individualisme forcené - un «égoïsme»-puisque, en l'absence de règles collectives suffisamment fortes et légitimes pour s'imposer à tous, les acteurs ne sont plus guidés que par la recherche individuelle et brutale du profit maximum ${ }^{40}$.

Dans cette première période de la pensée durkheimienne, le statut des émotions est donc clair. Les émotions individuelles sont essentiellement des états psychiques incompatibles avec l'ordre social. La régulation sociale doit donc avoir une double cible: l'individu et la société. Chez l'individu, elle doit lutter, par l'éducation, contre la désorganisation affective qu'engendre une émotivité non maîtrisée (Cf. L'Éducation morale). Au niveau de la société, elle doit permettre le renforcement, voire la création, d'un système de règles, afin que les rapports sociaux soient à la fois cohésifs et fonctionnels - pour que l'ordre social règne $(\mathrm{Cf}$. Le Socialisme de $1895^{41}$ et Le Suicide de 1897). Lorsque la société ne parvient pas ou plus à assurer cette régulation sociale, cette émotivité naturelle reprend le dessus: les individus perdent leur sens moral, retrouvent leurs vieux démons émotionnels, et les sociétés sont en proie à des épisodes destructeurs d'émotion, d'«effervescence » collective particulièrement néfastes.

\section{Phase 2: Les émotions pour l'Intégration}

Vers la fin des années 1890, un bouleversement va se produire dans la problématique durkheimienne de l'action sociale et de l'ordre social. Durkheim luimême en est parfaitement conscient. Dans une lettre célèbre, il écrit: «C'est seulement en 1895 que j'eus le sentiment net du rôle capital joué par la religion dans la vie sociale. [...] Ce fut pour moi une révélation. Ce cours de 1895 marque une ligne de démarcation dans le développement de ma pensée, si bien que toutes mes recherches antérieures durent être reprises à nouveaux frais pour être mises en harmonie avec ces vues nouvelles ${ }^{42}$. Cette «révélation» va conduire Durkheim à ne plus considérer l'ordre social sur le seul plan de la contrainte et de l'extério-

39 Philippe Besnard, L'Anomie. Ses usages et ses fonctions dans la discipline sociologique depuis Durkheim, Paris, P.U.F., 1987; Philippe Steiner, La Sociologie de Durkheim, Paris, La Découverte, 1994.

40 Émile Durkheim, De la division du travail social [1893], Paris, P.U.F., 1973.

41 Émile Durkheim, Le Socialisme: sa définition, ses débuts, la doctrine saint-simonienne, Paris, Retz-CEPL, 1978.

42 Émile Durkheim, Textes/1. Éléments d'une théorie sociale [1907], Paris, Minuit, 1975, p. 404. 
rité mais sur celui de l'adhésion à un monde symbolique. La régulation sociale n'est plus alors le maître mot de la sociologie durkheimienne. Les règles et les normes sociales, même intériorisées, ne suffisent plus à expliquer l'ordre social. Le second principe d'explication, celui de l'intégration sociale, va prendre alors une tout autre importance jusqu'à faire pencher définitivement la balance de son côté.

L'intégration sociale n'est plus seulement conçue comme l'intégration de l'individu dans la société, comme dans La Division du travail social ou dans Le Suicide où elle est surtout l'instrument par lequel la régulation sociale s'exerce sur l'individu en l'attachant au respect de normes. Elle devient bien davantage : la participation aux représentations collectives, le sentiment ressenti par l'individu d'une communion étroite entre lui et la société, jusqu'à ne pouvoir se définir luimême que par son appartenance à la société avec laquelle il ne fait qu'un. L'ordre social n'est plus essentiellement assuré par un principe externe de régulation mais par un principe interne à l'individu: ses conduites lui sont dictées non tant sous la pression sociale ou par des routines que par des convictions ${ }^{43}$.

L'acteur durkheimien ne se réduit donc plus essentiellement à un agent programmé et soumis. Cette autorité que possède la règle ne peut être que de nature sociale. La raison en est simple: seul ce qui nous «dépasse» peut exercer une emprise sur nous, et la seule chose qui nous dépasse c'est la société. Ainsi, la régulation des émotions et des conduites n'est possible que par l'obéissance aux règles morales. Mais comme ces règles sont de nature essentiellement sociale, cette obéissance n'est elle-même possible que si nous reconnaissons l'autorité de la société qui nous impose ces règles. C'est pour cela que toute éducation morale doit également viser - outre l'apprentissage de l' «esprit de discipline » - «l'attachement [de l'individu] aux groupes sociaux », c'est-à-dire à la valeur morale des règles sociales. Ici le ton de Durkheim n'est plus celui de la contrainte mais celui du désir. Nous ne pouvons en effet accepter de nous appliquer des règles contraignantes que dans la mesure où nous leur reconnaissons une légitimité, et cette légitimité est faite d'un mélange d'autorité et de désirabilité. Apprendre aux individus «l'attachement aux groupes sociaux » c'est donc leur démontrer rationnellement combien leur bonheur dépend de leur soumission à la morale collective et à ses règles. Durkheim insiste fortement sur ces deux faces de la règle morale, des normes sociales: «Dans un cas, [la société] est comme le Dieu jaloux et redouté, le législateur sévère qui ne permet pas que ses ordres soient transgressés; dans l'autre, c'est la divinité secourable, à laquelle le croyant se sacrifie avec joie.» ${ }^{44}$

Dans le langage weberien, il s'agit de passer du registre de l'action traditionnelle à l'action «normative» («rationnelle en valeur»): tandis que les routines de l'action «traditionnelle» sont morales sans raison, les conduites authentiquement morales sont librement et, donc, rationnellement choisies.

Tout ce que Durkheim tente de démontrer dans ce cours c'est que l'«éducation morale» peut être une éducation rationnelle d'un individu rationnel - et non, comme dans le cas de l'éducation religieuse d'antan, une éducation faisant appel

43 Voir: François Dubet, Durkheim sociologue de l'action: l'intégration entre le positivisme et l'éthique, in Cuin Charles-Henry (Éd.), op. cit., p. 203-221.

44 Émile Durkheim, L'Éducation morale, op. cit., p. 78. 
à l'irrationnel et conduisant à produire des individus passifs et routiniers. C'est toute la différence, selon lui, entre le «devoir» et le «bien»: «Le devoir c'est la morale en tant qu'elle ordonne et qu'elle prohibe; c'est la morale sévère et rude, aux prescriptions coercitives; c'est la consigne à laquelle il faut obéir. Le bien, c'est la moralité en tant qu'elle nous apparaît comme une chose bonne, comme un idéal aimé, auquel nous aspirons par un mouvement spontané de la volonté.» ${ }^{45}$

\section{Phase 3: L'essence émotionnelle de la vie sociale}

Si nous avons tenu à citer cette dernière phrase, c'est pour souligner son extrême ambiguïté. Qu'est-ce donc, en effet, qu'un «mouvement spontané de la volonté », sinon une formidable contradiction? La spontanéité, qui est inconsciente, mécanique, s'oppose à la volonté, c'est-à-dire à l'intentionnalité. Un mouvement est soit spontané soit intentionnel. Durkheim, pourtant, n'a pas l'habitude de jouer de l'ambiguïté, et encore moins celle de se contredire. En fait, comme nous allons le voir, la sociologie durkheimienne de l'action repose sur une conception de la rationalité qui n'est pas celle de la subjectivité de l'acteur mais celle de l'action - disons qu'elle repose sur un concept de rationalité objective et non de rationalité subjective.

On le comprendra mieux si l'on se réfère à l'évolution de la pensée durkheimienne. À partir de la fin des années 1890 , c'est-à-dire au milieu de sa carrière, Durkheim est taraudé par une question qui va orienter toute la seconde partie de sa réflexion et de son œuvre. Cette question est celle de l'autorité des normes morales, du fait qu'elles ne sont pas respectées seulement par crainte de sanctions mais bien en vertu même des sentiments de respect qu'elles rencontrent chez les individus. Il a bien conscience que l'explication par la socialisation est insuffisante: la seule intériorisation de ces normes ne permet pas de comprendre le véritable sentiment de respect qu'elles suscitent en nous.

Et Durkheim va d'autant moins tarder à trouver une réponse définitive à cette question que cette réponse était déjà inscrite en filigrane dans sa première sociologie. Pour le dire brièvement:

- Les individus n'acceptent de contrainte que dans la mesure où cette contrainte est d'origine sociale (Cf. La Division du travail social ou, mieux encore, Le Suicide);

- ce que Durkheim découvre peu à peu (en se mettant à la sociologie religieuse) c'est que cette acceptation découle d'expériences au cours desquelles ils prennent conscience que leurs conduites leur échappent, qu'ils ressentent des émotions qu'ils ne comprennent pas et qu'ils ont des conduites qu'ils n'ont pas délibérées (Cf. Les Formes élémentaires...) ${ }^{46}$;

- ces expériences sont d'un type particulier: ce sont des moments d' "effervescence collective» (superbement décrits dans Les Formes...);

45 Émile Durkheim, L'Éducation morale, op. cit., p. 79. (C'est moi qui souligne.)

46 Émile Durkheim, Les Formes élémentaires de la vie religieuse [1912], Paris, P.U.F., 1994, p. 295 sq. 
- les individus comprennent alors que la société a sur eux un pouvoir irrésistible, contre lequel il est vain de lutter;

- leur réaction normale est donc, faute de pouvoir résister à ce pouvoir, de se soumettre à lui - c'est-à-dire non seulement de lui obéir mais aussi de le respecter, de le sacraliser;

- or, l'essence de toute pensée religieuse est le sacré: d'où la définition durkheimienne du sentiment religieux comme un sentiment par lequel les individus reconnaissent à la société une puissance qu'ils vénèrent;

- par extension, tout ce qui vient de la société fait l'objet d'un sentiment sacré: le sacré est donc à la base de toute vie sociale ${ }^{47}$.

C'est ainsi que Durkheim résout la question qu'il s'était posée, dès 1906, dans le fameuse conférence sur «La détermination du fait moral». Cette question était: - Qu'est ce qui donne aux règles morales leur puissance; une puissance telle que, en général, elle n'a pas besoin de sanctions extérieures pour être maintenue; une puissance telle que les actes qui transgressent ces règles provoquent une réaction d'indignation? La réponse est: - C'est le fait que ces règles nous viennent de la société; parce que nous ressentons la société comme supérieure à nous (la société est terrible) et comme la source même de tout ce que nous sommes (la société est nourricière, protectrice, libératrice...); bref, parce que ce qui vient de la société a un caractère quasi sacré. «Ainsi, écrit Durkheim, vouloir la société, c'est, d'une part, vouloir quelque chose qui nous dépasse; mais c'est en même temps nous vouloir nous même. Nous ne pouvons vouloir sortir de la société, sans cesser d'être des hommes.» ${ }^{48}$

Cette expérience de la puissance à la fois terrible et bienfaisante de la société (c'est la définition même du sacré: ce que l'on craint et que l'on désire), nous la vivons en permanence - et toujours sur un mode émotionnel. C'est le cas lorsque nous participons à des manifestations collectives (c'est l'enthousiasme), lorsque nous sommes en présence de la personne d'autrui (c'est l'embarras, si bien étudié par Goffman), lorsque nous rencontrons certains symboles (c'est le respect), lorsque nous commettons un crime (c'est l'angoisse) ou lorsque nous en voyons commettre un (c'est l'indignation), etc.

Du coup, chez le second Durkheim, le statut sociologique des émotions se trouve considérablement transformé. Les émotions ne sont plus seulement des pulsions naturelles faisant obstacle à la vie sociale et qu'il faut contrôler de façon impitoyable. Certaines sont, au contraire, à l'origine même du sentiment religieux, c'est-à-dire du caractère sacré des représentation collectives et de la morale, c'est-à-dire de la vie sociale elle-même puisque celle-ci n'est possible que

47 R. Boudon montre qu'une lecture attentive des Formes permet d'identifier la sphère du «sacré» durkheimien et celle de ce que la sociologie contemporaine appelle les «valeurs »-ce qui lui permet d'affirmer que, loin d'être irrationnel, le sens du sacré relève de «bonnes raisons » (Raymond Boudon, 'Les formes élémentaires de la vie religieuse': une théorie toujours vivante, L'Année sociologique, 1999, vol. 49-1, p. 149-198).

48 Émile Durkheim, Détermination du fait moral [1906], Sociologie et philosophie, Paris, P.U.F., p. 75 . 
parce que nous respectons ses normes morales. C'est parce que la société suscite en nous certaines émotions collectives puissantes que, en retour, nous respectons ce qui vient d'elle. Et ce respect est lui-même, fondamentalement, producteur d'émotions ${ }^{49}$.

\section{Esquisse d'une théorie durkheimienne des émotions}

Il apparaît donc très clairement que l'action sociale durkheimienne n'est pas une action de type «traditionnel», routinière, ni même une action «normative» (rationnelle en valeurs) mais bien une action émotionnelle, une action «affectuelle».

Mais cette action émotionnelle est d'un type tout à fait particulier puisqu'elle est socialisée. Cela signifie que, si elle n'est pas intentionnelle, elle n'est pas non plus irrationnelle. Bien sûr, nous ne choisissons pas de préférer ce que nous respectons. Pour autant, nos choix sont rationnels, et ils le sont non pas parce que nous en délibérons rationnellement mais parce qu'ils sont objectivement les mieux adaptés à notre condition d'être humains, c'est-à-dire d'être sociaux. Comme le dit Durkheim: nous avons raison de «vouloir la société »! Dès lors, on comprend mieux la signification profonde de l'étonnante formule relevée tout à l'heure «un mouvement spontané de la volonté»: c'est bien spontanément que l'acteur durkheimien veut agir moralement! (Du même coup, l'action «rationnelle en finalité» de Weber, et plus encore l'action «utilitaire», se trouve exclue de la conception durkheimienne de l'action sociale: elle est caractéristique de l'acteur soit non socialisé, soit désocialisé en situation d'anomie collective.)

Le schéma suivant permet de spécifier le satut des émotions dans l'évolution de la réflexion durkheimienne, en identifiant (au regard de la typologie weberienne) les types de conduites auxquelles elles s'apparentent selon les formes de leur socialisation:

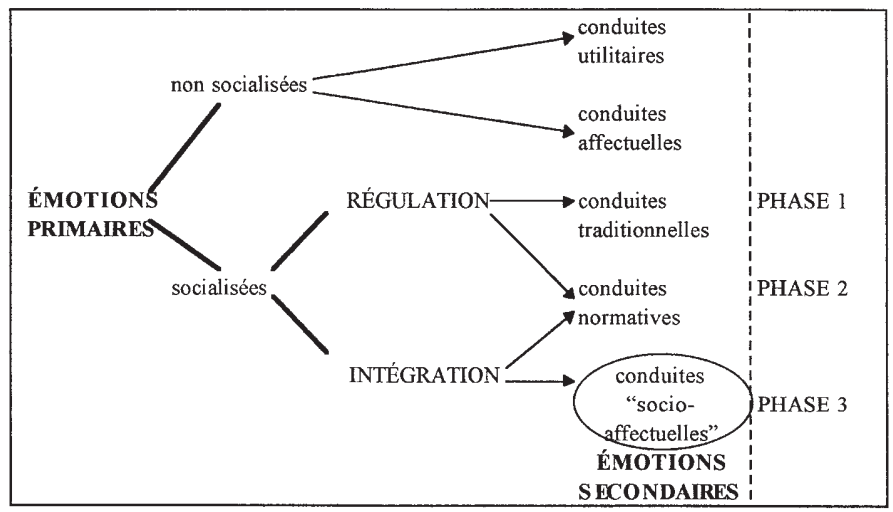

49 «Le respect est l'émotion que nous ressentons quand nous sentons cette pression intérieure et toute spirituelle se produire en nous. Ce qui nous détermine alors, ce ne sont pas les avantages ou les inconvénients de l'attitude qui nous est prescrite ou recommandée; c'est la façon dont nous nous représentons celui qui nous la recommande ou qui nous la prescrit.» (Émile Durkheim, Les Formes élémentaires de la vie religieuse, op. cit., p. 296.) 
Il est donc possible de reconstruire une véritable théorie des émotions chez Durkheim. Sous une forme stylisée, on pourrait l'expliciter comme suit:

- Il existe deux types d'émotions : primaires (ou «égoïstes») et secondaires (ou «altruistes»).

- Les premières sont celles qui se manifestent hors de tout contexte social ou bien lorsque la socialisation - «la société» - est défectueuse (petite enfance, périodes d'anomie).

- Les secondes sont celles qui naissent de l'expérience sociale elle-même - et, plus précisément, des moments où les premières se trouvent surexcitées par des situations sociales de dérèglement social (concentrations collectives, anomie profonde, périodes d' «effervescence $\left.{ }^{50} \ldots\right)$.

- Ces moments privilégiés sont producteurs de normes sociales qui ont d'autant plus d'emprise sur les individus que ces derniers leur confèrent un caractère de nature «sacrée»- nature au nom de laquelle ils les respectent.

- Ce respect est lui même de nature émotionnelle: le respect est en effet un sentiment spontané (non intentionnel).

- L'action sociale qui est à la base de la vie quotidienne en période normale est donc elle-même de nature émotionnelle - tout en possédant une forme particulière de rationalité, puisque les normes qui l'inspirent sont celles qui permettent à l'individu de vivre en société, ce qui est pour lui la seule manière d'être libre.

Ce qui, pour Durkheim serait objectivement irrationnel ce serait de se conduire soit de manière routinière («traditionnelle») soit de manière purement «utilitaire». Dans le premier cas, l'individu agirait sans savoir pourquoi; dans le second, il irait à l'encontre de ses intérêts d'être social - c'est-à-dire des seuls intérêts qu'il possède objectivement puisqu'il n'est rien d'autre qu'un être social.

Peut-être est-on alors en mesure de situer l'«action sociale» selon Durkheim dans le schéma typologique dont nous sommes partis. Pour notre part, c'est (presque) sans hésitation que nous situerions ce type d'action dans la case laissée vide (i.e., en haut et à droite) - c'est-à-dire comme une action à la fois rationnelle et non-intentionnelle. Il est en effet parfaitement «rationnel» non seulement de se soumettre à ce à quoi on ne peut pas se soustraire, mais aussi et surtout de respecter ce sans quoi nous serions «réduits à nos propres faiblesses » (sic) - ce qui, donc, nous constitue, nous est consubstantiel, nous confère notre humanité, ce qui nous libère en nous contraignant, ce qui nous donne notre identité. Bien sûr, ces raisons ne sont pas nécessairement subjectives (nous serions plutôt portés à penser que la société et ses normes nous diminuent, nous briment, nous frustrent). Ces raisons sont d'abord et essentiellement objectives: en voulant la société, nous nous voulons nous-mêmes et, ne pouvant pas ne pas nous vouloir nous-mêmes, nous ne

50 Philippe Steiner, Crise, effervescence sociale et socialisation, in Massimo Borlandi et Mohamed Cherkaoui (Éd.), Le suicide. Un siècle après Durkheim, Paris, P.U.F., 2000, p. 63-85. 
pouvons pas ne pas vouloir la société. L'action sociale durkheimienne est donc remarquable en ce qu'elle procède à la fois d'une «spontanéité » (les buts de l'action, nos «préférences » s'imposent à nous de façon quasi émotionnelle ${ }^{51}$ ) et d'une rationalité objective (nous avons raison de respecter les règles morales). Seulement, nous ne le savons pas vraiment: nous croyons généralement que cette morale est religieuse, ou bien que nous nous y conformons par utilité, ou bien encore qu'elle est «naturelle». Et il n'y aurait en fait que les sociologues qui le sauraient... ${ }^{52}$

\section{CONCLUSION: \\ LES ÉMOTIONS ET LE CHANGEMENT SOCIAL}

On vient de voir que, malgré des problématiques fort éloignées l'une de l'autre, nos deux auteurs s'accordaient non seulement pour attribuer aux émotions une place centrale dans l'analyse de l'ordre social, mais aussi - et c'est peut-être plus intéressant et surprenant encore - pour leur reconnaître un rôle déterminant dans le changement social.

Weber, pour sa part, cherche à comprendre comment, en dépit du caractère très largement émotionnel de l'action individuelle, les sociétés semblent évoluer inexorablement vers une rationalisation toujours plus forte. Pour l'essentiel, son explication réside dans la mise en évidence d'un processus décisif qui est celui de la «routinisation du charisme». Tendanciellement, c'est la rationalité normative, puis instrumentale, qui l'emporte sur l'affectivité. Le monde qu'il décrit est un monde froid ou, mieux encore, un monde qui ne cesse de se refroidir. Les charismes se routinisent, les sociétés se désenchantent, l'éthique de responsabilité supplante l'éthique de conviction ${ }^{53}$. Bref, selon son expression, «les dieux sont morts » et, avec eux, les démons que nous portons en nous. Si nos émotions continuent bien à nous agiter, il s'agit d'émotions «sécularisées»-d'émotions «sportives » (on pense ici à une phrase de L'Éthique protestante selon laquelle les Américains d'aujourd'hui pratiqueraient le capitalisme comme «un sport ${ }^{54}$ ). S'il fallait, dans ce domaine précis, lui désigner un successeur, ce serait évidemment Norbert Elias.

Durkheim, lui, voit les choses tout autrement. Après avoir longtemps pensé que la vie sociale n'était possible qu'au prix du contrôle des émotions qui nous ravalent au rang de primitifs ou d'animaux, il a réalisé que ces émotions sont aussi celles qui nous font adhérer à l'ordre social en nous révélant son existence, sa force, son «autorité». Il a donc compris que cet ordre social n'était possible que

51 «Au reste, si l'on appelle délire tout état dans lequel l'esprit ajoute aux données immédiates de l'intuition sensible et projette ses sentiments et ses impressions dans les choses, il n'y a peut-être pas de représentation collective qui, en un sens, ne soit délirante [...]» (Émile Durkheim, Les Formes élémentaires de la vie religieuse, op. cit., p. 325.)

52 «Tant que l'analyse scientifique n'est pas venue le lui apprendre, [1'homme] sent bien qu'il est agi, mais non par qui il est agi.» (Émile Durkheim, ibid., p. 299.)

53 Max Weber, «Le métier et la vocation de savant» [1919] in Max Weber, Le Savant et le politique, Paris, UGE, 1963.

54 Max Weber, L'Éthique protestante et l' esprit du capitalisme, op. cit., p. 250. 
parce que nous y croyions et, surtout, parce que nous y étions passionnément attachés. Allant encore plus loin, il a vu dans les émotions collectives le moteur même du changement social.

Et c'est sans doute sur ce point précis du rôle des émotions dans le changement social que Weber et Durkheim sont les plus proches. Tous deux estiment en effet que l'ordre social est produit et renouvelé par des émotions collectives puissantes, que cette activité émotionnelle est au principe même du changement social. Tous deux, en fin de compte, critiquent la rationalité poussée à son comble sous la forme de l'utilitarisme. Leur divergence essentielle réside dans le statut qu'il donnent à la rationalité: pour Weber, les raisons d'adhérer aux normes sont purement subjectives, et donc éminemment relatives; pour Durkheim ces raisons sont objectives, et donc moralement indiscutables. Weber est un relativiste, un chantre du «polythéisme des valeurs ${ }^{55}$; Durkheim est, de ce point de vue, un moraliste convaincu par sa morale, un religieux qui n'a pas besoin de Dieu.

En ce sens, ils sont bien, tous deux, des critiques romantiques des Lumières ${ }^{56}$ : les individus sont plus agis par des «passions» que par des «intérêts». Et, s'il s'accordent l'un et l'autre avec Nietzsche pour déclarer que «Dieu est mort», tous deux pensent qu'Il peut être remplacé: pour l'un (Weber) cela peut être par un nouveau charisme individuel, pour l'autre (Durkheim) c'est très certainement par ce charisme constamment renouvelé qu'est une société.

Nos deux auteurs sont donc, en fin de compte, aussi sentimentalement attachés aux émotions l'un que l'autre: l'un parce qu'il finit par les regretter, l'autre parce qu'il finit par les souhaiter ${ }^{57}$.

Département de sociologie-LAPSAC

Université Victor Segalen-Bordeaux II

55 Julien Freund, Sociologie de Max Weber, Paris, P.U.F., 1966.

56 Robert Nisbet, La Tradition sociologique [1966], Paris, P.U.F., 1984.

57 Pour terminer sur une note d'émotion, on rappellera que nos deux grands hommes sont morts l'un et l'autre assez jeunes, dans des circonstances bien différentes mais dans les deux cas fort cruelles - et qui ne sont pas sans intérêt: le théoricien du «désenchantement du monde» est mort de la grippe espagnole, tandis que le chantre (à la fin de sa vie) de l'exaltation de l'affectivité est mort de chagrin... 